\title{
Analysis of Denton E-Beam 2 Nanofabricated Thin Films by Metrology
}

\author{
Ronald Reliford Jr. ${ }^{1}$, and Jafar F. Al-Sharab ${ }^{1 *}$ \\ ${ }^{1 .}$ Department of Engineering Technology, Northwestern State University, Natchitoches, LA, USA. \\ * Corresponding author: jafar@nsula.edu
}

Instrumentation and metrology have been identified as critical nanotechnology areas and they are integral to the emerging nanotechnology era that we currently live. Instrumentation and metrology have a significant impact on various applications varying from electronics to medicine. Advances in nanofabrication, design of new nanomaterials, and ultimately manufacturing of new nanotechnologybased products will all depend to significantly on the capability to accurately and reproducibly measure properties and performance characteristics of the fabricated materials at the nanoscale. In this paper we are comparing results of freshly deposited chromium layer on silicon substrate using three different techniques, FESEM, Ellipsometry, and Profilometry.

The Denton E-Beam 2 (Denton Vacuum Infinity 22 electron beam evaporator) is a tool used for the physical vapor deposition of thin layer films and can be used in a vast variety of different thin layer film applications [1]. Use of the Denton E-Beam 2 in the CNST NanoFab at NIST allows users to access six different source materials at any one given time. This combined with the ability to perform depositions on multiple wafers of $150 \mathrm{~mm}$ makes this deposition method is a very popular tool. Different substrates are placed on the rotating dome of the tool so that a uniform deposition is given to each substrate. Automated recipe execution, uniformity, and versatility are all things that users can look forward to receiving when using this technique. The baseline procedure is executed on a monthly basis to ensure consistency and uniformity of the nanofabrication of thin films and deposits. In order to quantitatively analyze the results of the baseline procedure and ensure that the previously mentioned characteristics are present, advanced metrology tools are vital in this regard. In this research we employ Ellipsometry, Profilometry, and the SEM analysis tools to quantify the fabricated samples. The usage of multiple methods of metrology, is to investigate the consistency and uniformity of the fabricated films. In addition, results of this study will help identify standard, and reliable methods to measure surfaces at the nanoscale.

After a regulation-controlled deposition by E-Beam 2, Zeiss FESEM's with built-in analysis tool to perform a cross-section analysis of freshly deposited Cr layers on Si substrates with a layer of thermal oxide present. The analysis tool of the SEM allows for successful quantification of the thin films and will have its data recorded and used as a means of cross-checking against other methods of Metrology. Analysis using the Zeiss FESEM using the in-lens signal, after the baseline procedure, it was found that the Cr layer thickness is $32.75 \mathrm{~nm}$, Figure $1-\mathrm{a}$.

In the Ellipsometry technique, a 19-point map was performed. The Ellipsometer uses these 19 points to produce a datasheet which displays the average thickness present on the wafer's surface along with a statics sheet that shows each point's quantitative data. Using the Woollam Ellipsometer after the baseline procedure, it was found that an average layer thickness of $29.06 \mathrm{~nm}$, Figure 1-b. 
In this method, a very sensitive yet very precise stylus of the Dektak (Bruker Dektak XT) was used to measure the Cr layer thickness using the Dektak 1 Profilometer after performing the baseline procedure. Cr thickness using profilometry was found to be $27.78 \mathrm{~nm}$, Figure 1-c.

Using Ellipsometry, Profilometry, and the built-in analysis tool of the Zeiss FESEM we have confirmed the total layer thickness of the $\mathrm{Cr}$ deposited onto our baseline wafer to be between: $27.78 \mathrm{~nm}$ and $32.75 \mathrm{~nm}$. This means a mere $4.97 \mathrm{~nm}$ difference was found between our smallest and largest measured values. Using three separate methods of Metrology with correlating data such as the results we have produced gives us both confidence and certainty that the Denton E-Beam 2 has indeed produced an average $\mathrm{Cr}$ layer thickness of $29.86 \mathrm{~nm}$ after the baseline deposition process was executed. This is work on progress where statistical analysis will be used in the future [4].

\section{References:}

[1] A Wisitsoraat et al., Journal of Electroceramics 17(1) (2006), p. 45.

[2] H Fujiwara, Spectroscopic ellipsometry: principles and applications (John Wiley \& Sons).

[3] JM Alves et al., Review of Scientific Instruments 75(12) (2004), p. 5362.

[4] This work was conducted while the primary author an intern at NIST. He is very thankful to the National Science Foundation, NEATEC, and the National Institute of Standards and Technologies who allowed for the use of their resources to perform this research. Special thanks to Dr. Gerard Henein, process engineer at NIST, for providing exquisite training in the field of Nanotechnology.

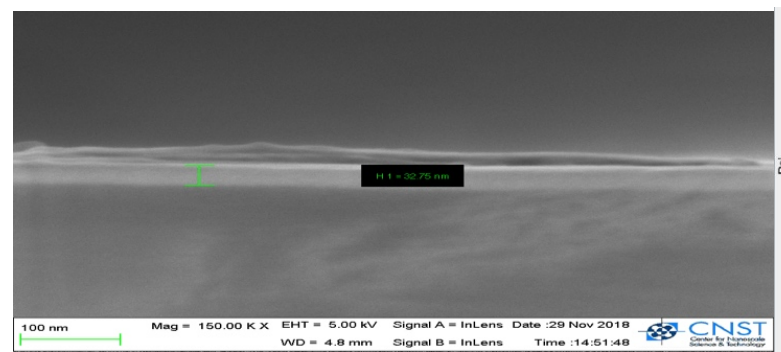

(a)

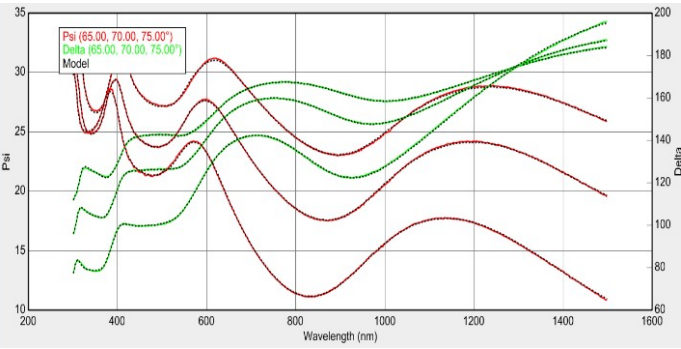

(b)

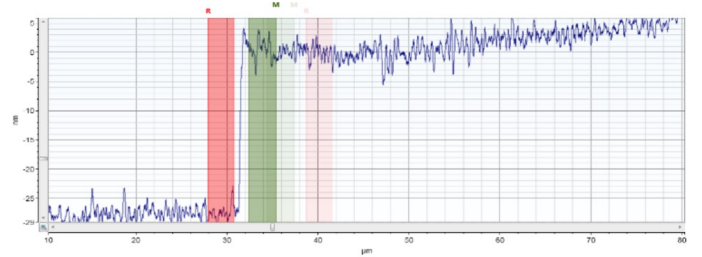

(c)

Figure 1. (a) Cross-section FE-SEM micrograph displaying the thickness of the deposited Cr layer, (b) Cr thickness analysis conducted by fitting to Chromium model mapped by Ellipsometry, (c) The output of a profilometer showing the thickness (step-up) of the Cr layer with respect to the Si substrate. 